\title{
Genetic variation in an isolated Italian population of fallow deer Dama dama as revealed by RAPD-PCR
}

\author{
Massimo SCANDURA, Ralph TIEDEMANN, \\ Marco APOLLONIO and Günther B. HARTL
}

\begin{abstract}
Scandura M., Tiedemann R., Apollonio M. and Hartl G. B. 1998. Genetic variation in an isolated Italian population of fallow deer Dama dama as revealed by RAPD-PCR. [In: Ecological genetics in mammals III. G. B. Hartl and J. Markowski, eds]. Acta Theriologica, Suppl. 5: 163-169.
\end{abstract}

Thirty-three random decanucleotide primers were tested for polymorphisms in a fenced Italian population of fallow deer Dama dama (Linnaeus, 1758), and 23 primers provided reproducible amplification patterns. The proportion of variable loci was $17.5 \%$ and the resulting intrapopulational similarity index was relatively high $\left(S_{a}=0.940\right)$. These data are in accordance with previous results obtained in screenings of other European fallow deer populations for biochemical variation. A comparison with a northern German specimen indicated low genetic variation also on an interpopulational level. Taking into account population histories (mass extinction, domestication, reintroductions) and the mating systems adopted, long-term effective population sizes in fallow deer should have been low, and low genetic variability could be expected. Given generally low levels of genetic variation, the RAPD-technique still detected a number of polymorphisms. Using a sufficient number of primers its resolution is considered satisfactory for individual typing or paternity analyses.

Dipartimento di Etologia, Ecologia, Evoluzione, Università di Pisa, via Volta 6, I-56126 Pisa, Italy (MS, MA); Institut für Haustierkunde, Christian-Albrechts-Universität zu Kiel, Olshausenstraße 40, D-24118 Kiel, Germany (MS, RT, GBH)

Key words: Dama dama, RAPD-PCR, genetic variability, genetic bottleneck

\section{Introduction}

Since the species became extinct in Europe during the last glaciation (Jarman 1972), none of the extant populations of fallow deer Dama dama (Linnaeus, 1758) present on this continent can be considered autochthonous. They are rather a result of repeated introductions of animals from their Pleistocene refugium in the Middle East, and of the subsequent establishment of many additional stocks using fallow deer originating from those founders (Ueckermann and Hansen 1983). In Italy, fallow deer is distributed in several isolated populations, with a total number of about 3000-4000 individuals (Apollonio, in press).

According to census data, there is no indication for these populations to be endangered. However, the subdivision into apparently isolated populations with small effective population sizes $\left(N_{\mathrm{e}}\right)$ is likely to be associated with a reduction of 
genetic variation due to random genetic drift and inbreeding. This effect may be enhanced by the mating system of fallow deer. In different populations, a variety of polygynous mating systems are adopted, which often result in an increase of the variance in male mating success. These include the lek, which is the most extreme polygynous mating system as yet known in ungulates (Randi and Apollonio 1988, Apollonio et al. 1989). Therefore, only a small proportion of males may actually participate in reproduction, causing a further reduction of $N_{\mathrm{e}}$ and an increase of the inbreeding level (Apollonio and Hartl 1993).

The Maremma Regional Park, in southern Tuscany, contains a wild fallow deer population, established in the first half of this century by subsequent introductions of individuals originating from a population in the San Rossore Preserve (Pisa, northern Tuscany). Within the Maremma Regional Park, a fenced population was formed by a modest number of animals captured between 1983 and 1990 from the wild population. At present, this population counts $150-160$ animals in a 42 ha area. The mating system of the fenced population represents the "dominance within groups"-system (Langbein and Thirgood 1989). Reproduction is concentrated between the last days of September and the first half of October. Before that time a hierarchy among males is established by means of roaring contents, threats, and occasionally by fights. Dominant males will monopolize the mates during the next breeding season, having exclusive access to receptive females throughout their estrous period. Occasionally, other males are tolerated in close proximity to them. Dominant males defend neither a territory nor resources.

Since the number of males participating in the matings is limited, $N_{\mathrm{e}}$ is expected to be particularly low and the inbreeding level rather high. In this framework, this paper presents data on the genetic variability of the fenced Maremma population, gained in the course of an assessment of the actual paternity of 56 calves born in the early nineties within the population. Since the utilized method of Random Amplified Polymorphic DNA-PCR (Williams et al. 1990) has as yet only rarely been applied to ungulates, we also aim at providing informations on the suitability of the technique and specific RAPD-primers for further genetic studies in cervids.

\section{Material and methods}

Whole blood and/or small pieces of ear tissue were collected from 25 adult individuals (22 males, 3 females) living in the fenced area in Maremma Regional Park. Additionally, one German specimen from Schleswig-Holstein was included in the analysis for comparison. Blood was diluted in EDTA. All samples were stored at $-20^{\circ} \mathrm{C}$ until genetic analysis. Genomic DNA was extracted from $2 \mathrm{ml}$ whole blood using the SuperQuikGene DNA isolation kit (Analytical Genetic Testing Center, Denver, USA) according to manufacturer's instructions. For DNA extraction from ear samples (usually $<10 \mathrm{mg}$ ) the Qiagen genomic DNA purification kit was used (Qiagen, Chatsworth, USA). The precipitated DNA was dissolved in a final volume of 100-200 $\mu \mathrm{l}$ TE buffer (10mM Tris, $1 \mathrm{mM}$ EDTA), and the concentration in each sample was quantified with a Hoefer TKO 100 fluorometer. Finally the DNA concentration was adjusted to $5 \mathrm{ng} / \mu \mathrm{l}$ by adding TE buffer. 
Table 1. Decanucleotides used in RAPD-amplifications.

\begin{tabular}{lccccc}
\hline Code & $5, \rightarrow 3$ & Code & $5{ }^{\prime} \rightarrow 3^{\prime}$ & Code & $5^{\prime} \rightarrow 3^{\prime}$ \\
\hline OPA-01 & CAGGCCCTTC & OPB-07 & GGTGACGCAG & OPB-18 & CCACAGCAGT \\
OPA-02 & TGCCGAGCTG & OPB-08 & GTCCACACGG & OPB-19 & ACCCCCGAAG \\
OPA-03 & AGTCAGCCAC & OPB-09 & TGGGGGACTC & OPB-20 & GGACCCTTAC \\
OPA-04 & AATCGGGCTG & OPB-10 & CTGCTGGGAC & OPC-01 & TTCGAGCCAG \\
OPA-05 & AGGGGTCTTG & OPB-11 & GTAGACCCGT & OPC-02 & GTGAGGCGTC \\
OPB-01 & GTTTCGCTCC & OPB-12 & CCTTGACGCA & OPC-03 & GGGGGTCTTT \\
OPB-02 & TGATCCCTGG & OPB-13 & TTCCCCCGCT & OPC-04 & CCGCATCTAC \\
OPB-03 & CATCCCCCTG & OPB-14 & TCCGCTCTGG & OPC-05 & GATGACCGCC \\
OPB-04 & GGACTGGAGT & OPB-15 & GGAGGGTGTT & GHO-01 & GCCGTCCGAG \\
OPB-05 & TGCGCCCTTC & OPB-16 & TTTGCCCGGA & GHO-02 & CAGCCTCGGC \\
OPB-06 & TGCTCTGCCC & OPB-17 & AGGGAACGAG & GHO-03 & GGGACGTCTC \\
\hline
\end{tabular}

RAPD-PCR was performed using 33 decamers commercially available (Operon Technologies, Alameda, USA; see Table 1). Taking into consideration different attempts to optimize the RAPD-technique (Riedy et al. 1992, Yu and Pauls 1992, Levitan and Grosberg 1993, Micheli et al. 1994, Bielawski et al. 1995), a modified protocol was developed. PCR conditions were $0.2 \mathrm{mM}$ dNTPs, 1.5 $\mathrm{mM} \mathrm{MgCl}$, $10 \mathrm{mM}$ Tris- $\mathrm{HCl}, 50 \mathrm{mM} \mathrm{KCl}, 0.5$ units Taq Polymerase (Appligene, Illkirch, France), 5 pmol of primer and 5-10 ng of genomic DNA in a total volume of $10 \mu \mathrm{l}$. Amplification parameters were an initial denaturation at $94^{\circ} \mathrm{C}$ for $1 \mathrm{~min}$, followed by 45 cycles of $1 \mathrm{~min}$ at $94^{\circ} \mathrm{C}, 1 \mathrm{~min}$ at $45^{\circ} \mathrm{C}$ and $2 \mathrm{~min}$ at $72^{\circ} \mathrm{C}$. PCR products were electrophoresed on a $2 \%$ agarose gel $(1.2 \%$ Metaphor, $0.8 \%$ NuSieve, FMC, Rockland, USA), and visualized with UV-light in the presence of ethidium bromide. Note, that only a random subset of the samples was tested with each primer ( $\mathrm{cf} n \mathrm{I}$ in Table 2).

Pairwise similarity was calculated for each pair of individuals and for each primer using the band sharing index $S_{\mathrm{AB}}=2 n_{\mathrm{AB}} /\left(n_{\mathrm{A}}+n_{\mathrm{B}}\right)$, where $n_{\mathrm{AB}}$ is the number of fragments shared by individuals $A$ and $B$, and $n_{\mathrm{A}}$ and $n_{\mathrm{B}}$ are the respective number of fragments scored in each individual (Lynch 1990). This index is mathematically identical to a similarity index described by Dice (1945) and also applied in RAPD data analysis (eg Comincini et al. 1996). Mean pairwise similarity $(S)$ as well as standard errors were calculated for each primer using the jackknife procedure (Efron 1982). Mean similarity over all primers $\left(S_{\mathrm{a}}\right)$ was weighted by sample size.

\section{Results}

Twenty five out of the 33 primers tested were included in the final analysis, their selection based on the quality and reproducibility of the amplification. These primers produced a total of 98 clear and reproducible amplification products (bands). The size of the amplificates ranged from 300 to 2000 base pairs. Altogether 19 bands (amplified with 13 different primers) were polymorphic (present/absent). Assuming that each band with a characteristic molecular weight represents one locus, and that bands amplified by different primers also represent distinct loci, the proportion of polymorphic loci was $19.4 \%$ over all samples, and $17.5 \%$ for the Italian population only. The mean similarities for single primers within the Italian population ranged from $S=0.819$ to $S=1$ (Table 2). The mean similarity over all 
Table 2. Similarity among individuals of the Italian population: $n \mathrm{I}$ - number of individuals, $n \mathrm{~L}-$ number of loci, $P$ - percentage of polymorphic loci, $S$ - mean similarity index \pm SE. Additionally, mean pairwise similarity between the Italian individuals and the German specimen are given $\left(S_{\mathrm{D}}\right)$. $S$ and $S_{\mathrm{D}}$ were compared with a $t$-test (ns $-p>0.05,{ }^{*}-p<0.05$, ** $-p<0.01$, *** $-p<0.001$ ). $S$ and $S_{\mathrm{D}}$ were calculated by the jackknife procedure (Efron 1982) apart from \# were only one value was available due to limited sample size.

\begin{tabular}{lrrrccc}
\hline Primer & $n \mathrm{I}$ & $n \mathrm{~L}$ & $P$ & $S$ & $S_{\mathrm{D}}$ & $p$ \\
\hline OPA-01 & 6 & 3 & 0 & $1.000 \pm 0.000$ & $0.800 \pm 0.000$ & $0.0000 * * *$ \\
OPA-04 & 4 & 7 & 29 & $0.916 \pm 0.016$ & $0.958 \pm 0.024$ & $0.1986 \mathrm{~ns}$ \\
OPA-05 & 4 & 7 & 14 & $0.962 \pm 0.013$ & $0.981 \pm 0.019$ & $0.4468 \mathrm{~ns}$ \\
OPB-01 & 22 & 6 & 67 & $0.819 \pm 0.001$ & $0.866 \pm 0.021$ & $0.0315^{*}$ \\
OPB-03 & 3 & 3 & 33 & $0.867 \pm 0.067$ & - & - \\
OPB-05 & 5 & 1 & 0 & $1.000 \pm 0.000$ & - & - \\
OPB-08 & 5 & 4 & 0 & $1.000 \pm 0.000$ & - & - \\
OPB-09 & 5 & 4 & 0 & $1.000 \pm 0.000$ & - & - \\
OPB-10 & 3 & 2 & 0 & $1.000 \pm 0.000$ & - & - \\
OPB-12 & 11 & 7 & 43 & $0.893 \pm 0.002$ & - & - \\
OPB-13 & 7 & 5 & 0 & $1.000 \pm 0.000$ & - & - \\
OPB-14 & 2 & 4 & 25 & $0.889 \#$ & - \\
OPB-15 & 6 & 4 & 25 & $0.954 \pm 0.011$ & $0.976 \pm 0.024$ & $0.4119 \mathrm{~ns}$ \\
OPB-16 & 3 & 3 & 33 & $0.867 \pm 0.067$ & - & - \\
OPB-17 & 3 & 3 & 0 & $1.000 \pm 0.000$ & - & - \\
OPB-18 & 4 & 3 & 33 & $0.900 \pm 0.033$ & - & - \\
OPB-19 & 3 & 1 & 0 & $1.000 \pm 0.000$ & - & - \\
OPB-20 & 4 & 2 & 0 & $1.000 \pm 0.000$ & $1.000 \pm 0.000$ & $1.0000 \mathrm{~ns}$ \\
OPC-01 & 4 & 6 & 17 & $0.955 \pm 0.015$ & $0.822 \pm 0.022$ & $0.0026 * *$ \\
OPC-03 & 4 & 3 & 0 & $1.000 \pm 0.000$ & - & - \\
OPC-04 & 4 & 6 & 17 & $0.955 \pm 0.015$ & $0.977 \pm 0.023$ & $0.4517 \mathrm{~ns}$ \\
OPC-05 & 4 & 3 & 0 & $1.000 \pm 0.000$ & $0.800 \pm 0.000$ & $0.0000 * * *$ \\
GHO-01 & 7 & 4 & 0 & $1.000 \pm 0.000$ & - & - \\
GHO-02 & 5 & 5 & 0 & $1.000 \pm 0.000$ & - & - \\
GHO-03 & 3 & 1 & 0 & $1.000 \pm 0.000$ & & - \\
\hline & & & & & & - \\
\end{tabular}

25 primers was $S_{\mathrm{a}}=0.940$. When the mean similarity within the Italian population $S_{\mathrm{a}}$ and the mean pairwise similarity between the Italian individuals and the German specimen $S_{\mathrm{D}}$ were compared, 5 out of 9 primers did not reveal any significant difference. Three primers indicated that the German specimen was genetically less similar to the Italian individuals than the Italian individuals to one another, while in one primer the German specimen had a significantly higher mean similarity. The statistical significance of the latter, however, was weak (Table 2). Mean pairwise similarity over 9 primers between the German specimen and the Italian individuals was $S_{\mathrm{a}}=0.896$, while the similarity among Italian specimens was $S_{\mathrm{a}}=0.912$ for the same set of primers. 


\section{Discussion}

Since each RAPD-investigation is based on its own particular primer set and population genetic studies aimed at estimating genetic variability are as yet quite scarce, any decision upon a particular level of genetic similarity detected to be indicative of genetic depletion is difficult. However, when our data are compared with the results of similar studies, which, even if not on the same set of loci scored, is based on the same basic statistics used in the present paper, there are some indications that our population of fallow deer is genetically quite homogeneous.

The proportion of polymorphic loci within the Italian population was $17.5 \%$ (17/97), and mean similarity was $S_{\mathrm{a}}=0.940$. The latter value is comparable to results of a RAPD-study carried out on three other local Italian cervid populations (Comincini et al. 1996): $S_{\mathrm{a}}=0.896$ for fallow deer from Bosco della Mesola (Ferrara), $S_{\mathrm{a}}=0.961$ for red deer Cervus elaphus from the same location, and $S_{\mathrm{a}}$ $=0.972$ for roe deer Capreolus capreolus from Bosco di Carrega (Parma). However, in each species the similarity value was based only on 4 specimens and 8 primers and, thus, can be considered only approximate. In a more thorough RAPD analysis on three Japanese populations of Sika deer Cervus nippon, within population similarity indices were $S_{\mathrm{a}}=0.89, S_{\mathrm{a}}=0.84$, and $S_{\mathrm{a}}=0.97$, respectively (Tamate et al. 1995). Here, the last $S_{\mathrm{a}}$-value was interpreted as indicative of genetic homogeneity of the respective population (Kinkazan). On the contrary, Chung et al. (1995) found $74 \%$ of 78 RAPD-bands to be polymorphic in Holstein domestic cattle Bos taurus $\mathrm{f}$. domestica with a mean similarity of $S_{\mathrm{a}}=0.353$. Even if they are not directly comparable, the difference among these values of genetic variability and those obtained for deer populations are substantial.

If one accepts our results as being indicative of a high homogeneity among individuals in the fenced Maremma population, this study would represent an additional case of a European fallow deer population showing a low level of genetic variability. Previous studies already revealed low levels of biochemical polymorphisms in various European populations: Hartl et al. (1986) studied a fenced population in southern Germany and detected polymorphism at only one out of 15 protein loci examined by horizontal starch gel electrophoresis. This locus, coding for the enzyme catalase, was found polymorphic also by Randi and Apollonio (1988), who screened 51 loci in an Italian population of fallow deer. The mean proportion of polymorphic loci $(P)$ in these studies ranged between 0 and 0.066 , while the highest value of average heterozygosity was 0.018 . The low levels of genetic variation in local fallow deer populations are in accordance with expectations derived from general population history of European fallow deer (see Introduction) and the mating systems found in this species (Apollonio and Hartl 1993).

In addition to the apparent genetic homogeneity within populations of fallow deer, there are also indications for genetic similarity on an interpopulation level: Pemberton and Smith (1985) screened 30 enzyme loci in fallow deer from 37 populations in England and Wales and did not find any variation within or among 
localities. This is corroborated by our comparison between Italian individuals and the German specimen showing also a high pairwise similarity of 0.896. Though only based on a single German individual, this might be a first indication of a large-scale homogeneity among European populations. For the British populations, Pemberton and Smith (1985) hypothesized the occurrence of a bottleneck either at the time when fallow deer was introduced to Britain or, alternatively, before such introduction, presumably during the last glaciation. However, to confirm the validity of the latter assumption on an European scale, more thorough investigations are needed, including also the Middle East population representing a presumable source for all the extant European populations.

Given a far reaching genetic homogeneity in fallow deer at the population, if not at the species level, we like to emphasize the potential resolution of the RAPD-technique applied. Even though the Italian population was genetically quite similar to a Northern German specimen, significant additional similarity arose within the population. And - despite of this intrapopulation similarity - quite a number of polymorphisms was present, which, if based on a sufficient number of primers, makes RAPD a potential tool even for individual typing, ie in the course of paternity testing.

Acknowledgements: We are grateful to A. Chines, S. Giannelli, P. Gigantesco, R. Mantovani and I. Rossi for the collection of samples. We also thank G. Brandtmann for critical comments on a preliminary version of this manuscript.

\section{References}

Apollonio M., Festa-Bianchet M. and Mari F. 1989. Correlates of copulatory success in a fallow deer lek. Behavioral Ecology and Sociobiology 25: 89-97.

Apollonio M. and Hartl G. B. 1993. Are biochemical-genetic variation and mating systems related in large mammals? Acta Theriologica 38: 175-185.

Apollonio M. (in press). [Fallow deer]. [In: La Fauna d'Italia, Mammiferi, Ungulati e Carnivori. L. Boitani, S. Lovari and A. Vigna-Taglianti, eds]. Calderini, Bologna. [In Italian]

Bielawski J. P., Noack K. and Pumo D. E. 1995. Reproducible amplification of RAPD markers from vertebrate DNA. Biotechniques 18: 856-860.

Chung E. R., Kim W. T. and Han S. K. 1995. Analysis of DNA polymorphisms and genetic characteristics in Holstein dairy cattle using RAPD-PCR technique. Korean Journal of Animal Science 37: 455-466.

Comincini S., Sironi M., Bandi C., Giunta C., Rubini M. and Fontana F. 1996. RAPD analysis of systematic relationships among the Cervidae. Heredity 76: 215-221.

Dice L. R. 1945. Measures of the amount of ecologic association between species. Ecology 26: 297-302.

Efron B. 1982. The jackknife, the bootstrap, and other resampling plans. Society for industrial and applied mathematics, Philadelphia: 1-92.

Hartl G. B., Schleger A. and Slowak M. 1986. Genetic variability in fallow deer, Dama dama L. Animal Genetics 17: 335-341.

Jarman M. R. 1972. European deer economies and the advent of the Neolithic. [In: Papers in Economic Prehistory. E. S. Higgs, ed]. Cambridge University Press, Cambridge: 125-147.

Langbein J. and Thirgood S. J. 1989. Variation in mating systems of fallow deer (Dama dama) in relation to ecology. Ethology 83: 195-214. 
Levitan D. R. and Grosberg R. K. 1993. The analysis of paternity and maternity in the marine hydrozoan Hydractinia symbiolongicarpus using randomly amplified polymorphic DNA (RAPD) markers. Molecular Ecology 2: 315-326.

Lynch M. 1990. The similarity index and the DNA fingerprinting. Molecular Biology and Evolution 7: $478-485$.

Micheli M. R., Bova R., Pascale E. and D'Ambrosio E. 1994. Reproducible DNA fingerprinting with the random amplified polymorphic DNA (RAPD) method. Nucleic Acids Research 22: 1921-1922.

Pemberton J. M. and Smith R. H. 1985. Lack of biochemical polymorphism in British Fallow deer. Heredity 55: 199-207.

Randi E. and Apollonio M. 1988. Low biochemical variability in European fallow deer (Dama dama L.): natural bottlenecks and the effects of domestication. Heredity 61: 405-410.

Riedy M. F., Hamilton W. J. and Aquadro C. F. 1992. Excess of non-parental bands in offspring from known primate pedigrees assayed using RAPD-PCR. Nucleic Acids Research 20: 918.

Tamate H. B., Shibata K., Tsuchiya T. and Ohtaishi N. 1995. Assessment of genetic variations within populations of Sika deer in Japan by analysis of randomly amplified polymorphic DNA (RAPD). Zoological Science 12: 669-673.

Ueckermann E. and Hansen P. 1983. Das Damwild. Paul Parey, Hamburg und Berlin.

Williams J. G. K., Kubelik A. R., Livak K. J., Rafalski J. A. and Tingey S. V. 1990. DNA polymorphisms amplified by arbitrary primers are useful as genetic markers. Nucleic Acids Research 18: 6531-6535.

Yu K. and Pauls K. P. 1992. Optimization of the PCR program for RAPD analysis. Nucleic Acids Research 20: 2606.

Received 23 March 1998, accepted 30 April 1998. 